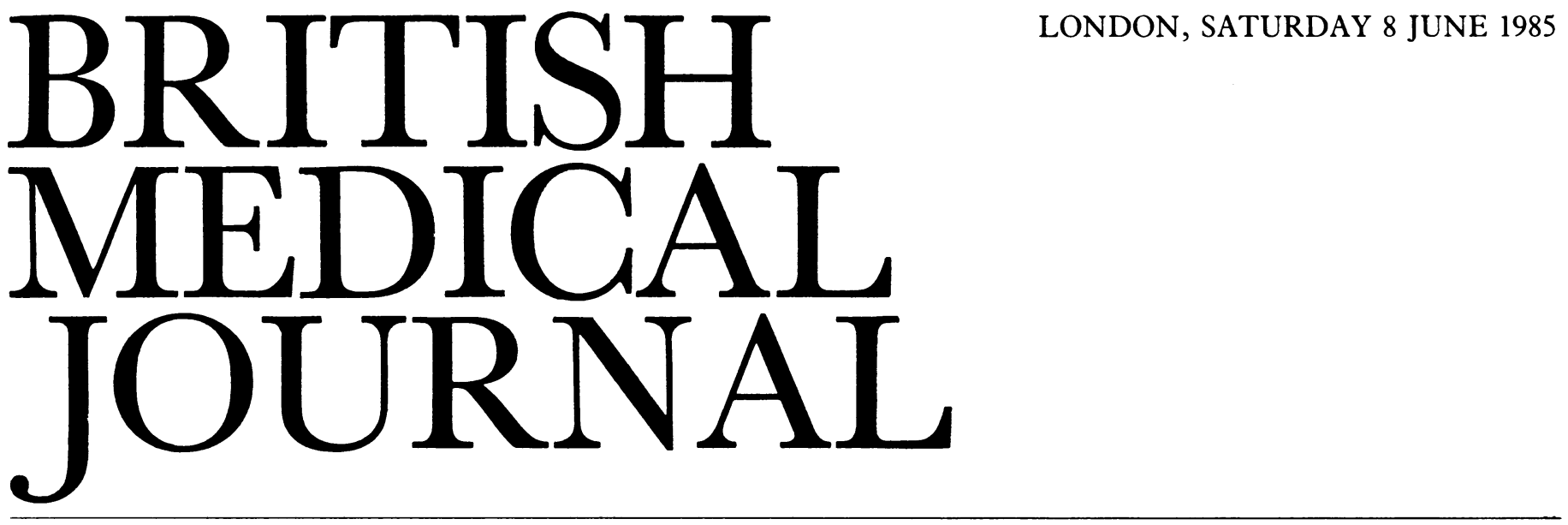

\title{
The political argument on health costs
}

An important part of the present government's policy is substantially to reduce public expenditure. Since spending on the National Health Service makes up about one eighth of public expenditure it is unlikely to be exempted from this general political strategy. Health care is emotive, however, and the NHS popular. It is, therefore, politically inexpedient to cut the service too drastically - or, at least, to be seen to do this. Hence any government wishing to reduce public expenditure faces a dilemma with the NHS - which is already remarkably cheap. Indeed, Britain spends less per head on health care resources than other developed countries. ${ }^{1}$

One way out of the dilemma is for politicians to try to cut services and at the same time try to maintain a public impression of improving resources. At the most glib political level any government's commitment to the NHS is measured by comparing expenditure on an annual basis adjusted only for inflation. At this level, if politicians can simply and truthfully claim that real expenditure in any year is increased they can rebut any suspicion of cutting services. Moreover, other statistics may be selected to carry particular messages, as, for instance, was recently shown in an education policy debate.

In reality the assessment of health policy is more complicated. The data required to judge a government's political commitment to the NHS are, firstly, real expenditure related to need; secondly, improvement in outcome consequent on this expenditure; and, thirdly, some measure of patient satisfaction-as well as health worker satisfaction. So we need to know by how much changes in expenditure are attributable to changes in salaries or conditions of employment, to industrial action, and to changes in efficiency, as well as to demographic changes among many other things. For example, the largest real annual increase in NHS expenditure in the past 10 years was mostly attributable to unusual increases in salaries.

Changes in real expenditure should be judged, therefore, by relating expenditure on medical services at constant prices explicitly to population growth and population aging. This would be but a first step because within age groups illness rates themselves vary-but it is clearly a necessary first step.

To seek to compare outcomes, special studies have to be carried out on effectiveness, efficiency, and acceptability of care. The net effect of medical progress is to increase requirements for resources for constant levels of medical need. Year by year it has become possible to treat effectively certain patients who previously could not be treated or to increase the effectiveness of treatment; and the extra expenditure required is judged to be justified by improved outcome. Even devolving care to the community implies increased expenditure from another branch of the public purse. It is, indeed, relatively unusual for medical progress to give rise to a reduction in expenditure for a given outcome. Effective medical technology can usually command a price which reflects the implied value of the consequence of using it rather than the cost of development and manufacture. In medical care the implied value of improving outcome is often very high indeed, and thus it is more difficult to constrain costs-though this should be easier in a centralised system than a free market system because budgets are inflexible.

None of this analysis is novel-which makes it the more surprising that inadequate summaries of crude indices of NHS performance are apparently thought to be enough for the public, patients, and people who work in the NHS to judge the implied priority given to health. Politicians should be aware that we understand (as well as they do) that increases in real expenditure do not necessarily imply any real increase relative to changing need and medical advances. Nor, therefore, do they imply increasing consumer satisfaction.

Recently, the government has taken to producing press releases showing the "Latest figures on health service activity," and in April community health councils, family practitioner committees, and others around the country received half a million pamphlets entitled The Health Service in England, based on the first press release. ${ }^{5}$ These documents are clearly designed to persuade their recipients of the government's high priority for the NHS. It may be true, but these documents do not go very far towards proving it. They contain selected ${ }^{6}$ tables and figures showing changes in various indices between 1978 and 1983 and compare these with changes in the same indices between 1973 and 1978 .

Consider two instances. Firstly, the number of inpatient cases (this is a misnomer because they are episodes not cases) increased by nearly 5\% between 1973 and 1978 and by over $12 \%$ between 1978 and 1983. This kind of statistic typifies the presentation which receives wide publicity. But what does it mean? Does it mean that inpatient services are more effective? Does it mean that hospital provision is more efficient than it was before 1978 and that everybody is working much harder? Does it even mean that the availability of effective and acceptable standards of health care is greater than it was? 
Such data may imply none of these things, but clearly these are some of the questions to be addressed-yet they are not. For example, these data could easily be presented in relation to population size, and yet the documents contain hardly a single population based rate. We cannot, therefore, tell how much of the increase is simply due to the increasing proportion of elderly people in the population. The necessary calculation is not complex-indeed, something very similar is performed routinely to compare resources for regional health authorities with the Resource Allocation Working Party's (RAWP) formula. The method is not perfect but it is a good deal better than counting episodes of admission. We are now used to seeing seasonally adjusted unemployment figures; we might reasonably expect population adjusted expenditure and utilisation figures.

Likewise, we are told, the volume of coronary bypass surgery has more than doubled since 1978. But in 1978 the evidence for its efficacy was less certain than it is now. What are the resource consequences of this increase? How many eligible patients remain untreated in spite of it, judged perhaps by numbers on waiting lists in given years? What is the effect of changing length of stay on overall costs and therefore aggregate efficiency? What does it mean for the staff of busier wards? What is happening to morbidity and mortality from coronary heart disease? Without answers to these questions the data have little meaning.

The collection of NHS statistics depends on the good will and effort of people who work in the NHS, and the Department of Health and Social Security should recognise its obligation to collate and report the data in a manner which is meaningful and useful. Only when this is done can the people who work for the NHS recognise the service they know in the descriptions produced by politicians. As it is, most people are left with an uneasy feeling that either they are unusually badly off or that statistics are meaningless. They need not be.

I am grateful to Phil Strong, Alison McFarlane, Angela Coulter, and Ann $\mathrm{McPherson}$ for helpful comments.

\section{KLIM MCPHERSON}

Lecturer in Medical Statistics,

Department of Community Medicine and General Practice,

Radcliffe Infirmary,

Oxford OX2 6HF

1 Office of Health Economics. Compendium of health statistics. 5th ed. London: Office of Health Economics, 1984:table 2.2

2 Flather P. Fall-out from Oxford. Newi Statesman 1985 Mar 15:10

3 Office of Health Economics. Compendium of health statistics. 5th ed. London: Office of Health Economics, 198 :

4 Department of Health and Social Security. Latest figures on health service activity. London Department of Health and Social Security, 1984. Press release No 844420,28 Dec 1984.

Fowler N. The health seritice in England. London: Department of Health and Social Security, 1985

6 Radical Statistics Health Group. Unsafe in their hands. London: Radical Statistics Health Group. 1985

\section{Non-cemented hip prostheses}

In Britain 38000 total hip replacements are performed each year, ' but the problem of permanently securing the artificial joint to bone remains. ${ }^{23}$ Acrylic cement provides excellent immediate fixation, but unfortunately the effect does not always last and painful loosening of the prosthesis is the most important long term complication of total hip replacement. ${ }^{45}$ Between four and nine years after total hip replacement 20-
$50 \%$ of femoral prostheses show radiological evidence of loosening, ${ }^{67}$ and in patients aged 30 or less $57 \%$ develop radiolucent lines over $2.5 \mathrm{~mm}$ wide at the bone-cement interface, or movement of the prosthesis, or require further surgery within five years. ${ }^{8}$ Revision surgery is often technically demanding, and the results are inferior to those of a primary replacement of the hip.

Loosening of the prosthesis may be due to a combination of factors. Firstly, in overweight or extremely active patients the forces transmitted through the artificial joint exceed the strength of its fixation, which may result in "mechanical" loosening. Secondly, the strength of the supporting layer of acrylic cement may deteriorate with time and repeated weight loading. ${ }^{9}$ Cement is not a true adhesive (there is no known substance which will permanently adhere to bone $)^{10}$ and acts simply as a grouting agent, filling irregularities between the endosteal surface of the bone and the prosthesis and thus holding the two together. It has a crystalline structure so that cracks may propagate in areas under excessive stress. ${ }^{9}$ Thirdly, bone next to the cement may undergo resorption leaving only a fibrous membrane, evident radiologically as a lucent line which may expand with time." This "biological" loosening may be a thermal, chemical, or allogeneic response by the bone attempting to isolate a prosthesis, which it recognises as a foreign body. Alternatively, it may represent disuse porosis - for example, in the proximal femur.

The mechanisms of loosening are not well understood, but fracture of the cement, giant cell reactions to wear and tear debris, and remodelling of bone all play a part. Moreover, the fibrous membrane at the bone-cement interface of loose joints is not inert for it produces prostaglandin $E_{2}$ and collagenase and these may cause further resorption of bone. ${ }^{12}$ Infection at the bone-cement interface accelerates resorption of bone and loosening of the prosthesis. In vitro studies suggest that methylmethacrylate cement may inhibit the chemotactic migration of polymorphonuclear leucocytes towards bacteria such as Staphylococcus epidermidis, Staph aureus, and Escherichia coli, ${ }^{13}$ as well as inhibiting the phagocytic and killing properties of these cells. ${ }^{14}$

All these adverse local effects of acrylic cement are long term problems, but it may also cause systemic effects at the time of surgery. Intraoperative, cardiovascular, and pulmonary complications have tended to occur at the time of impaction of the femoral component, and some have attributed this to absorption of toxic acrylic monomers. ${ }^{15}$ In rabbits methylmethacrylate is known to be cardiotoxic. ${ }^{16}$ Adverse reactions in man include cardiac irregularities, arterial hypotension (which is rarely fatal), and arterial hypoxaemia. ${ }^{17}$ Alternative explanations of these complications include pulmonary fat embolism, ${ }^{18}{ }^{19}$ pulmonary microembolism, ${ }^{20}$ neurogenic reflexes, ${ }^{21}$ and air embolism. ${ }^{22}$

Given the adverse effects of cement and given that it acts simply as a grouting agent between the prosthesis and the bone, why not dispense with it and use a carefully designed prosthesis which fits securely into an accurately cut bone? Numerous non-cemented hip replacements have already been developed, principally on the Continent, and in Russia, where cement is not widely available, non-cemented prostheses predominate. ${ }^{23}$ It was in Britain, however (where cement fixation was first widely used), that the complications of its use were first pointed out and the earliest reports of cementless fixation appeared. ${ }^{24}$

Studies in animals have attempted to establish the ideal overall size, shape, and surface texture of a prosthesis which would ensure intimate bone-prosthetic contact and encourage 